\title{
SPECULAR OBJECTS IN RANGE CAMERAS: REDUCING AMBIGUITIES BY MOTION
}

\author{
Jonas Nygårds and Åke Wernersson ${ }^{+}$ \\ Robotics / Autonomous Mechanical Systems \\ Linköping University S - 58183 Linköping SWEDEN \\ email:jonny@ikpgw.liu.se fax: int.46-13281101 \\ ${ }^{\dagger}$ Also with Luleå University
}

Abstract: Range cameras using structured light and triangulation are essentially based on the assumption of one diffuse reflection from the measured surfaces. Specular and transparent objects usually give multiple reflections and direct triangulation can give different types of 'ghosts' in the range images. These 'ghosts' are likely to cause serious errors during gripping operations.

As the robot moves some of the 'ghosts' moves in an inconsistent way. In this paper, we study, experimentally and theoretically, how the range measurements can be integrated in a consistent way during the motion of the robot.

Emphasis is on parts with 'optical complications' including multiple scattering.

For a scene with one planar mirror the 'ghosts' are shown to lie in a plane separated from the laser plane. In this case the orientation and position of the mirror can be estimated.

Keywords: Range camera, sheet of light, specular reflections, transparent objects, sensor feedback for gripping, active reduction of ambiguities.

\section{INTRODUCTION - THE PROBLEM}

For autonomous gripping and navigation, range cameras are useful geometric sensors. This paper is towards understanding their basic limitations. Emphasis is on mirror like objects as in Fig. 1, the ambiguities generated by single reflections in a mirror can be reduced when the robot is moving.

The paper starts by describing experiments that illuminate the problems and also justifies the forthcoming analysis.

The motion of the robot can be used to detect and estimate the orientation of the mirror in Fig. 1.

Also, a new type of holographic lens is presented. This lens, gives 19 equally spaced dots on a line instead of the usual plane, thus reducing the possibility of ambiguities.

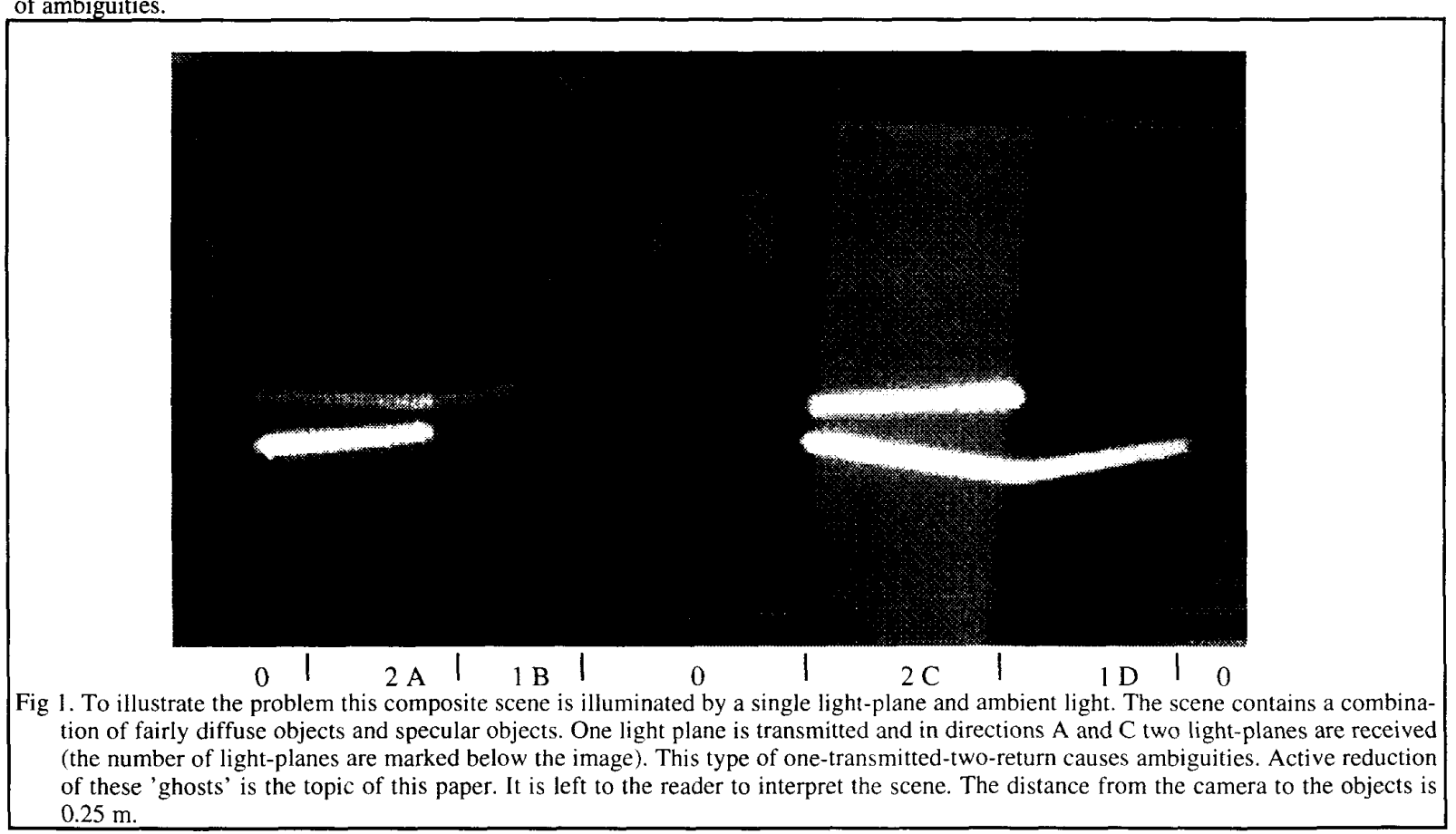

This work was partially funded by NUTEK(J.N.) and faculty sponsored by CENIIT

0-7803-2072-7/94\$4.00@ 1994 IEEE 
The treatment of transparent objects is also simplified since each ray can be traced.

The following analysis is purely geometrical for the laser plane-camera combination, with one mirror in the scene. For a photometric analysis of specularities during motion see [ZGB89] and [Bla85]. Occlusion is not studied in this paper.

\section{A Recalling the Principles of the Range Camera based on light-plane projection} Fig 2.

The basic principle of the range camera is illustrated in

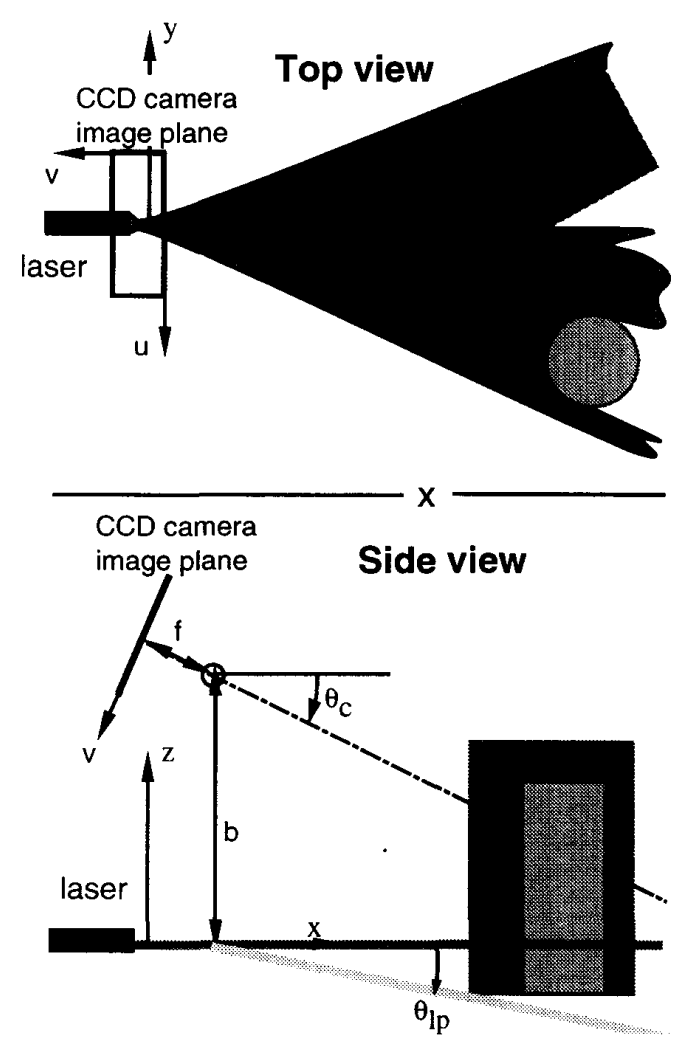

Fig 2. Notations for a range camera using sheet of light illumination. Only a pinhole model [Ho86] is used. To obtain a full 3D range picture the light plane will be deflected from the standard orientation by the angles $\theta_{l p} \in\left[\theta_{\min ,}, \theta_{\max }\right]$ as illustrated with the light grey plane touching the bottom of the box. The direction of one individual ray within the plane $P$ is then defined by the two angles $\theta_{l p}$ and $\beta$

For the intersection between a light-plane ray with angle $\beta$ and the scene, the $(x, y, z)$ coordinates are found by triangulation.

The camera parameters are:

$b$ - distance between the camera and the light plane

$\theta_{c}$ - orientation of the camera

$f$ - focal distance and scaling of the $(u, v)$-axes, (assumed to be known from calibration).

In the normal case of diffuse reflection (Lambertian model [NIK91], [Ho86]), the intensity $I(u, v)$ detected by the camera emanates from the intersection between the light plane $P$ and the object. Triangulation is used to calculate the intersections between $P$ and the rays going through the pinhole of the camera collecting the intensity at $(u, v)$.

This gives the range and direction to all objects reflecting the intensity of the plane and within the view of the camera. However, not all intensity collected by the sensor array is emanating from the plane $P$.

\section{TYPICAL IMAGES FOR SPECULAR AND TRANSPARENT OBJECTS}

The range camera principle above basically assumes a diffuse (Lambertian) surface. This section and the introductory Fig 1 illustrates that many common materials generates images that are far from being Lambertian. For photometric models of specular reflection see [NIK91] or [Wo194].

\section{A Experiments for a Glass Bottle}

In Fig. 3, the laser intensity image for a sheet-of-light illuminated bottle is shown.

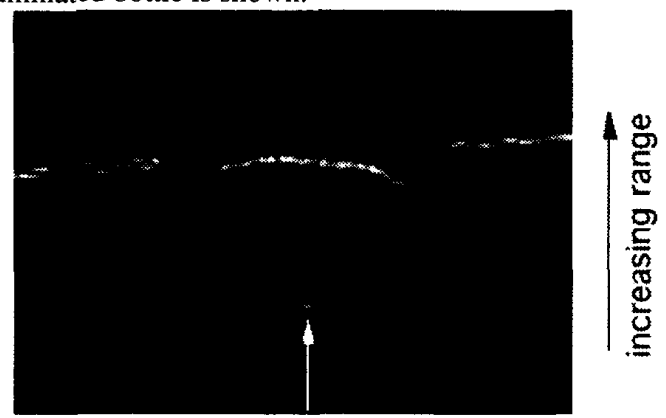

Fig 3. A glass bottle with a paper label on the rear side. The bottle is laying on a flat surface and illuminated by a sheet of light. The flat surface is clearly visible and the label is seen through the bottle as the bright 'concave' curve. The 'convex' front side of the bottle is barely visible due to the transparency. The blob indicated by the white arrow has an angle close to specular incidence.

The laser intensity image is obtained by taking the difference between one image with the laser on and a second without the light-plane. The transparent glass reflects some light since the angle is close to the specular. The paper label is brighter since the Lambertian surface reflects more light in the direction of the camera. Only the specular spike on the glass closest to the viewer, is as bright as the paper.

This case shows that to be able to handle transparent objects multiple responses of varying intensity in different directions must be considered. 


\section{B. Experiments for a Partially Specular Surface}

The coffee cup in Fig 4 is made of a ceramic material. Ceramics are dielectrics and show specular reflection at high incidence angles (shown by Wolff, [Wol94]). In Fig. 4 effects of the specular reflection can be seen on the flat surface behind the cup. Beneath the straight line from the light plane/surface intersection, a weaker slightly bent line appears at the arrows. This line is generated by light with high incidence angle reflected by the curved surface at both sides of the cup.

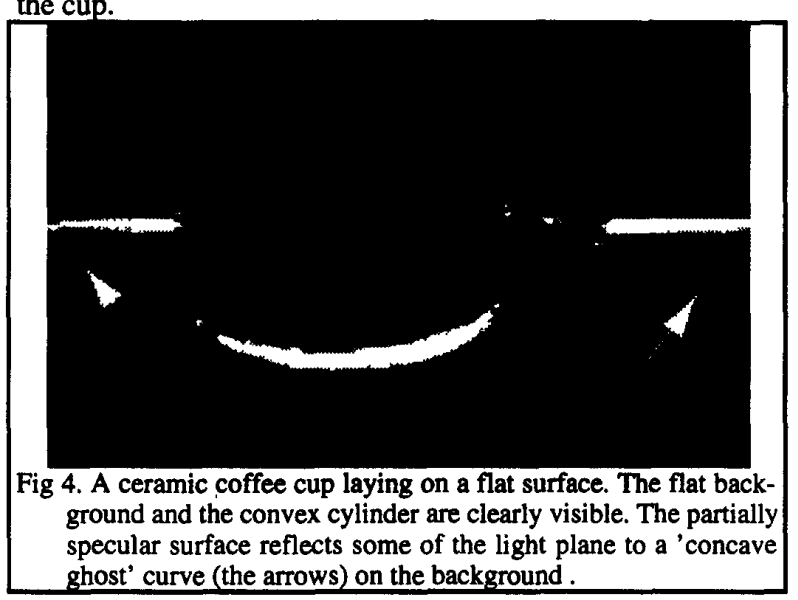

In this case, the direct triangulated image would create an impression of a semi transparent, slightly curved surface in front of the flat background. For a polished mirror surface the 'concave ghost' would have been much brighter than the true convex cylinder intersection.

\section{Range Image for an Object with Internal Scattering (Nylon Bolt)}

In this example the light-plane is scattered inside the material causing a wide diffuse line. In the image in Fig 5 the laser line on the bolt is much more diffuse than the line on the paper box. This causes high variance when the v-coordinate in the image is extracted using a centre of mass calculation.

\section{Different Types of Scattering}

From above and other examples, different sources of light are identified in the image:

- Diffuse scattering (Lambertian object, background in Fig 3 and Fig 4 )

- Transparent objects which also have specular peaks (the bottle in Fig 3)

- Specular scattering (specular object Fig 1, 3 and 4)

- Second order scattering (Fig 1)

- Internal scattering (Fig 5)

- Diffuse scatter from within the light-plane-generating lens [NW93]
- Diffraction (In Transparent objects. Not exemplified.) Experienced in some unpublished experiments using a Fresnel lens.

- Scattering in many small objects (not exemplified).

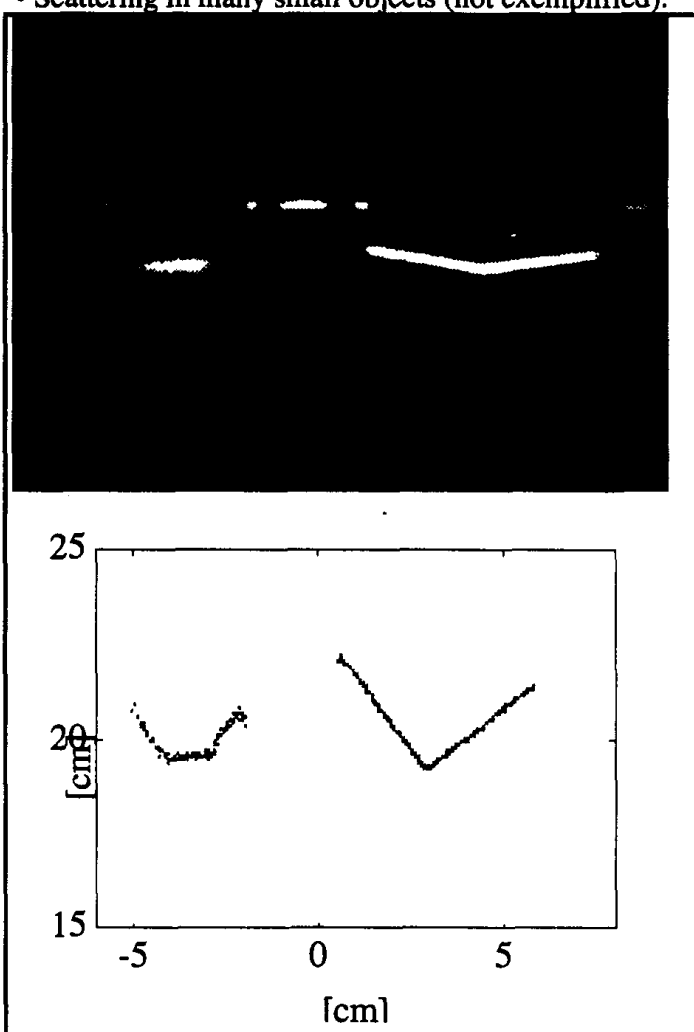

Fig 5. Camera image (top) and extracted range (bottom) of a scene consisting of an nylon bolt head to the left and a paper box as a reference to the right. Notice how the bolt head is almost glowing, giving increased range noise.

\section{EXPERIMENTS WITH MULTIPLE SCATTERING AND MOTION.}

Let us return to the initial scene in Fig 1. This scene consists of one paper box of dimensions $34 \times 35 \times 85 \mathrm{~mm}$ and, to the left of the box, a metal mirror of dimensions $44 \times 204 \mathrm{~mm}$ tilted backwards $\theta_{m}=4.3$ degrees. Three range photos were taken of this scene at distances 0.100 .20 and $0.30 \mathrm{~m}$ away from the box (Fig 6). Between the image acquisition the motion was along the light-plane keeping $z \equiv 0$.

In the range plots in Fig 6 we can see two corners which are consistent with the cameras ego motion. The corner to the right is the true corner of the box whereas the corner to the left is a range mirror image.

The cause of the non moving corner is the following: The light-plane is reflected back by the mirror and illuminates the rear side of the paper box. This illuminated corner is then reflected by the mirror to the camera. This false corner turns out to be stationary as the robot approaches the scene. 


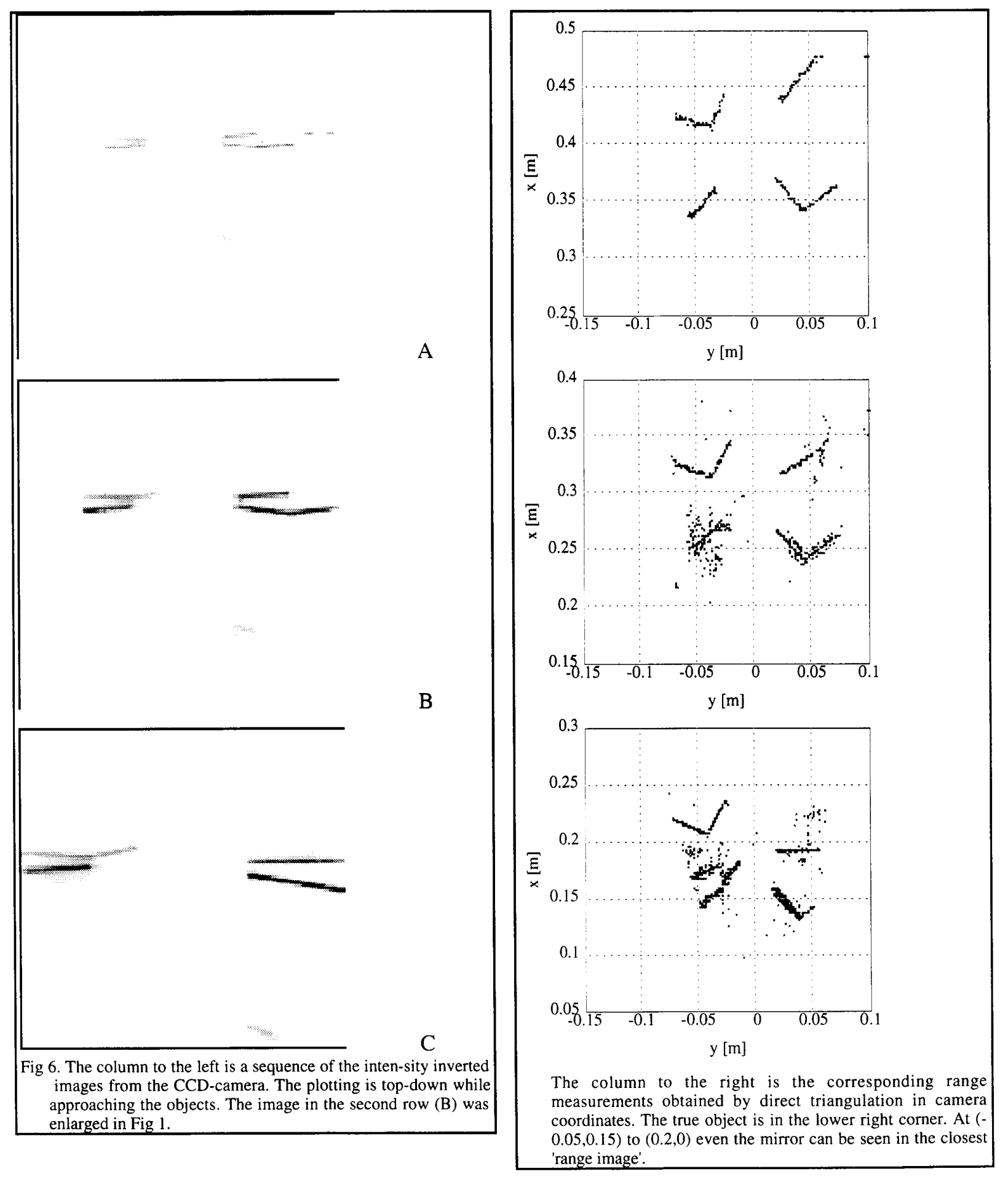


The line above the corner to the right is the closest of the false intersections. In the range pictures it can be noted how this line and the mirror image of the true light-plane/box intersection line are moving towards the boxes. This motion is clearly detectable and indicates a possible mirror in the scene.

The range measures were made using a low threshold for distances 0.100 .20 to see as much reflections as possible in spite of the noise. By this low thresholding it is possible to see the intersection light-plane/mirror in the $0.1 \mathrm{~m}$ image corresponding to the line between $(x=0.15, y=-0.05)$ and $(x=0.2, y=0)$ in the range plot. Using a proportional low threshold for the $0.3 \mathrm{~m}$ distance introduces too much noise. By the use of a higher threshold the $0.3 \mathrm{~m}$ range image is less noisy than the other two.

\section{ANALYSIS FOR SCENES CONTAINING ONE PLANE MIRROR}

Consider a scene with one planar mirror as in Fig. 1. Four cases for a ray to propagate from the laser to the camera are: case O: $\quad$ laser $\rightarrow$ Object $\rightarrow$ camera

case MO: laser $\rightarrow$ Mirror $\rightarrow$ Object $\rightarrow$ camera

case OM: laser $\rightarrow$ Object $\rightarrow$ Mirror $\rightarrow$ camera

case MOM: laser $\rightarrow$ Mirror $\rightarrow$ Object $\rightarrow$ Mirror $\rightarrow$ camera

The basic range camera is thus case $O$.

For completeness two more cases are mentioned: case OO: laser $\rightarrow$ Object $\rightarrow$ Object $\rightarrow$ camera case OMO: laser $\rightarrow$ Object $\rightarrow$ Mirror $\rightarrow$ Object $\rightarrow$ camera

Case $\mathrm{OO}$ is the well known mutual illumination. Both are excluded since they generally do not produce any significant intensity in the range image.

\section{A. Properties of the illuminating planes}

To specify the illuminating light plane, Fig. 2 and Fig 7, we use the position of the light-plane focus:

$L_{0}=\left[\begin{array}{lll}x_{L 0} & y_{L 0} & z_{L 0}\end{array}\right]^{T}$

and the surface normal :

$\hat{n}_{p}=\left[\begin{array}{lll}\sin \left(\theta_{l p}\right), & 0, \cos \left(\theta_{l p}\right)\end{array}\right]^{T}$

Let $\beta$ be the angle along the light-plane. The direction unit rays of the light-plane can then be written:

$P=\left\{\left[\cos (\beta) \cos \left(\theta_{i p}\right), \quad \sin (\beta), \quad-\cos (\beta) \sin \left(\theta_{i p}\right)\right]^{T}\right\}$

with $\beta \in\left[\beta_{\min }, \beta_{\max }\right]$ as the angle along the scan.

By tracing the individual rays of $P$ through a scene as in section 3 the three mirror cases MO, OM and MOM are investigated for a flat mirror.

By this it is shown that:

- The virtual position of collected light of case MOM lies on the extension of the ray emitted in that direction. The 'ghost' behaves just as a true object at the virtual position.

- Emitted light of both cases MO and OM belong to the same plane. If the mirror normal is not orthogonal to the light plane normal, points belonging to this reflected plane will be outside the light-plane. When the camera is moved their motion will be inconsistent. This is the case for the two moving lines in section 3 .

For the scenes illustrated in Fig. 1, 6 and 7 there are, conceptually, four planes of light.

Two real planes (in 'front' of the mirror):

Plane $P$ : The plane transmitted by the cylindrical lens at the laser. This plane contains all illumination for the standard case $\mathrm{O}$.

Plane $\boldsymbol{P}_{\boldsymbol{g}}$ : When the transmitted plane $\boldsymbol{P}$ hits the mirror, a second plane, $P_{g}$ is generated by reflection in the mirror. This plane gives the illumination for all MO cases. Fig. 7A shows the two illuminating planes.

\section{Two virtual planes ('behind' the mirror):}

Plane $\boldsymbol{P}_{\boldsymbol{v}}$ :The transmitted plane $\boldsymbol{P}$ illuminates objects in the scene. When these illuminated objects are observed through the mirror they are described to be illuminated by the virtual plane $P_{\nu}$. In this plane the OM cases is found (Fig 7B).

Plane $\boldsymbol{P}_{\boldsymbol{v}}$ : The plane generated by the mirror $\boldsymbol{P}_{\boldsymbol{g}}$ illuminates objects in the scene. When these illuminated objects are observed through the mirror they are described to be illuminated by the virtual plane $P_{v g}$. Plane $P_{v g}$ finally contain the MOM cases (Fig. 7C).

For these four planes the following properties hold

Property 1: The two planes $P_{g}$ and $P_{v}$ are identical with surface normal :

$\hat{n}_{g}=\hat{n}_{v}=\hat{n}_{p}-2\left(\hat{n}_{p} \bullet \hat{n}_{M}\right) \hat{n}_{M}$

(proof in appendix).

Property 2 directly follows from the expression of the surface normal above

Property 2: If $\hat{n}_{p} \bullet \hat{n}_{M} \neq 0$ then both mirror-images (OM) and the reflected-laser-intersections (MO) lie in a plane outside the original light-plane, $P$. As the robot moves, a range image based on the assumption that light only emanates from plane $P$ is inconsistent with a stationary scene.

The detection of this inconsistency depends on the distance between the 'ghost' and the light-plane (a larger distance is easier to detect).

Property 3: The two planes $P$ and $P_{v g}$ are identical with surface normal $\hat{n}_{v g}=\hat{n}_{P}$ (proof in appendix).

As a consequence, 'ghosts' of type MOM belong to plane $P$ and will move consistently with the robot in the range image.

Property 4: If the motion of the robot is confined to the light-plane, the planes $P, P_{v}$ and $P_{g}$ remain unchanged (except for their extensions). All objects intersecting with these planes can only be illuminated at this intersection during motion.

Proof: If the robot moves within the light-plane, the new light-plane will be equal to the old (except for their extensions). Since all the other planes only depend on the laser generated light plane the geometry will remain unchanged. However, the distribution of rays (the intensity) within the planes changes. 


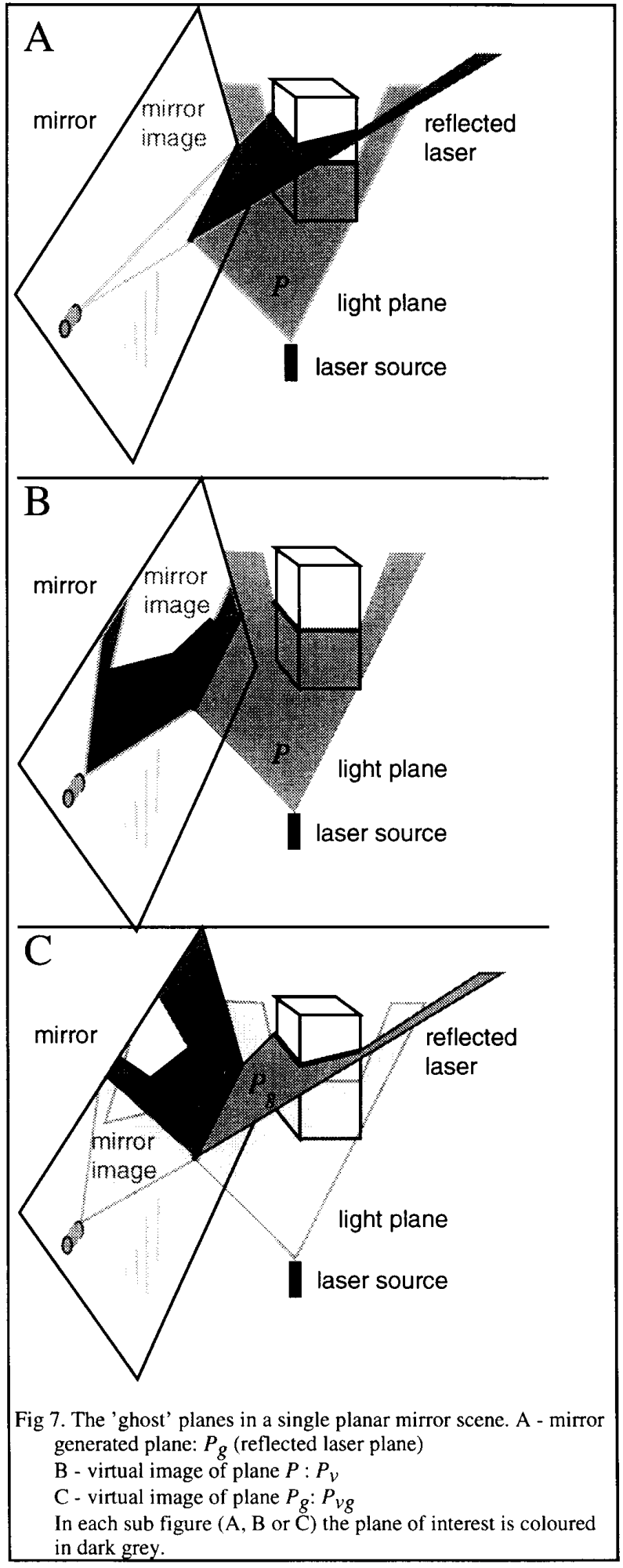

\section{V. 'CHASING GHOSTS' USING ACTIVE ILLUMINATION AND MOTION}

If the motion of the range camera (camera and laser) is known, some of the 'ghosts' above can be detected and excluded.

The sheet of light defines a plane $(z=0)$ in the $(x, y, z)$ space and the robot is assumed to have its motion in that plane.

In this section, equations for estimating the true $3 \mathrm{D}$ position from 2D range images during motion are given. Basically the idea is to use the optic flow ([Ho 86] and [DaX91] ) of the 'ghosts' to determine their 3D-location. Since we expect to find range cameras on the market with only range data as output, the calculations will be in the range image instead of the ordinary camera image. This solution will have the same shortcomings as other solutions to optic flow (aperture problems, correspondence problems etc.).

The correspondence problem will be simplified for scenes with only one mirror. Only points not corresponding to the transmitted light plane need to be considered.

\section{A. Using Motion to Localise Range 'ghost' in $3 D$ from $2 D$ - Light Plane Detections}

If the camera collects large intensities emanating from points outside the light plane (described in section 3 ) the solution for the intersection with the light plane will obviously give a false position.

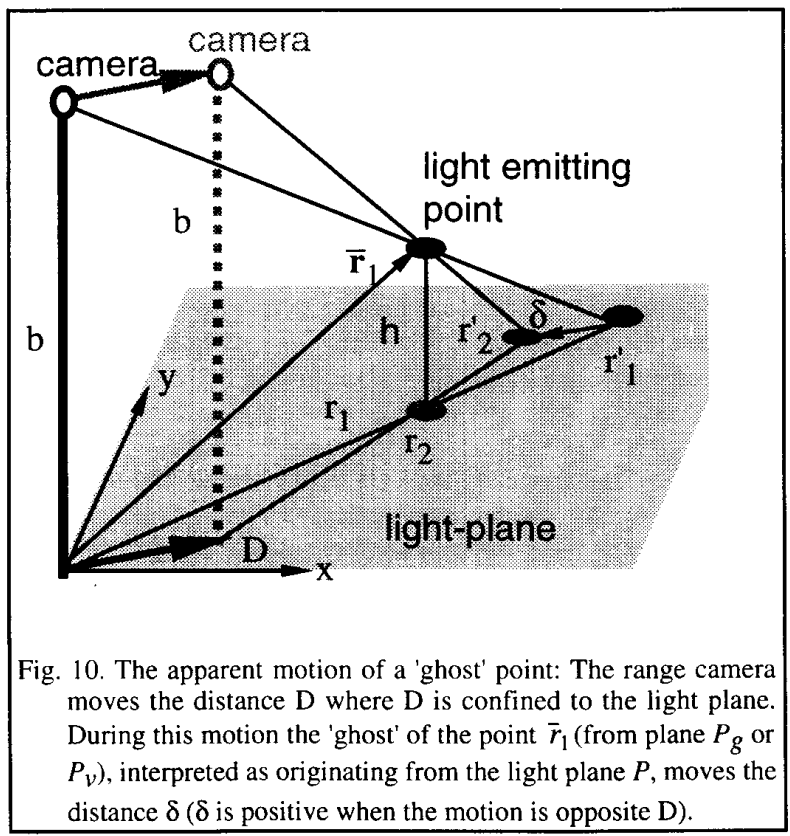


Since the motion and the geometry of the sensor is known, the true 3D-position can be estimated from a range image sequence of 'ghosts' confined to the light plane.

As a consequence of property 4 above the true position of all 'ghosts' are stationary in the scene.

Consider one well defined point shaped 'ghost' at height $h$ outside the light plane ( $\bar{r}_{1}$ in Fig. 10).

$$
\bar{r}_{1}=\left[\begin{array}{lll}
r_{1} \cos \left(\alpha_{1}\right) & r_{1} \sin \left(\alpha_{1}\right) & h
\end{array}\right]^{T}
$$

with $\alpha_{1}$ as the angle between the $x$-axis and the projection of $\bar{r}_{1}$ on the $x-y$-plane (light-plane).

The range camera image of this 'ghost', will be at the position $r_{1}$, where the light plane is intersected by the line passing through the camera pin hole and the 'ghost'. When the camera is moved, the range camera image of this point will move in a revealing way.

From the geometry in Fig 10 we get the following uniform triangle equations:

$$
\left.\begin{array}{l}
\frac{b-h}{r_{1}}=\frac{b}{r_{1}^{\prime}} ; \frac{b-h}{r_{2}}=\frac{b}{r_{2}^{\prime}} \\
\frac{D}{r_{2}}=\frac{\delta}{r_{2}^{\prime}-r_{2}} ; \frac{D}{r_{1}}=\frac{\delta}{r_{1}^{\prime}-r_{1}}
\end{array}\right\}
$$

Now the true 3D coordinates of the point as seen from the first scanning position can be expressed using, the first measurements of the 'ghost' $\left(r_{l}^{\prime}\right.$ and $\left.\alpha_{1}\right)$, its detected motion $(\delta)$ and the known camera motion (D).

$$
\bar{r}_{1}=\left[\frac{D}{D+\delta} r_{1}^{\prime} \cos \left(\alpha_{1}\right), \quad \frac{D}{D+\delta} r^{\prime}{ }_{1} \sin \left(\alpha_{1}\right), \quad \frac{b \delta}{D+\delta}\right]^{T}
$$

For the detection of a 'ghost', its apparent motion $\delta$ must be significantly larger than the combined uncertainty of the range-camera and the motion of the camera.

From the equation above it can be seen that the detection of 'ghosts' will depend on their distance $h$ from the light plane and the step length $\mathrm{D}$ since:

$$
h=\frac{b \delta}{D+\delta} \Rightarrow \delta=\frac{D h}{b-h}
$$

In a scene with only one mirror, property 1 above show that the 'ghosts' will all belong to the same plane $\left(P_{g}=P_{\nu}\right)$. If the detected 'ghosts' span this plane, a least squares method can be used to optimally fit a plane to the detected 'ghosts' as in [AcH88]. (Using the results in the appendix also cylindrical mirrors can be treated in a similar manner.)

Knowing the plane $P_{g}$ we can now estimate the plane of the mirror by the intersection line between the reflected lightplane and the original light-plane and the mirror normal vector

$n_{M}=\left(\hat{n}_{P}+\hat{n}_{g}\right) \times\left(\hat{n}_{P} \times \hat{n}_{g}\right)$

\section{B. Finding the Mirror in Experimental Data of Multiple Scattering and Motion}

Returning to the experiment in section 3, we use equations (6), (7) and (9) to estimate the position of the mirror.

From the range-images from each position, the end points of the 'ghost lines' (marked with ' $x$ ' in Fig 11) were manually selected. The true 3D positions of these 'ghosts' were esti- mated (projections on $x-y$-plane marked with 'o' in Fig 11). From their 3D position the orientation and position of the mirror was estimated as the solid line in Fig 11.

Currently, work is being done using innovation based detection [MP 71], [BSF 88] to automatically extract the 'ghosts' [KLO93].

Note that the angle of the mirrored corner is not rectilinear since it is produced by the reflected light plane cutting the box at an angle of $2 \theta_{m}$ to the x-y plane.

As illustrated by the upper 'o'-pair in Fig. 11 the position of the mirror image of the box will not coincide with this corner.

Since the box is standing almost upright, the projection of the reflected laser-box intersection has almost the same $x-y$ coordinates as the box / light plane intersection (as shown by the lower 'o'-pair).

Note also the effect of using the centre of mass for the position of the detected laser line. The intensity of the mirror intersection line is merging with the line of the reflected light-plane intersecting the box. At the lower left end of the points belonging to the mirror, this is causing the small bias towards the 'ghost line'

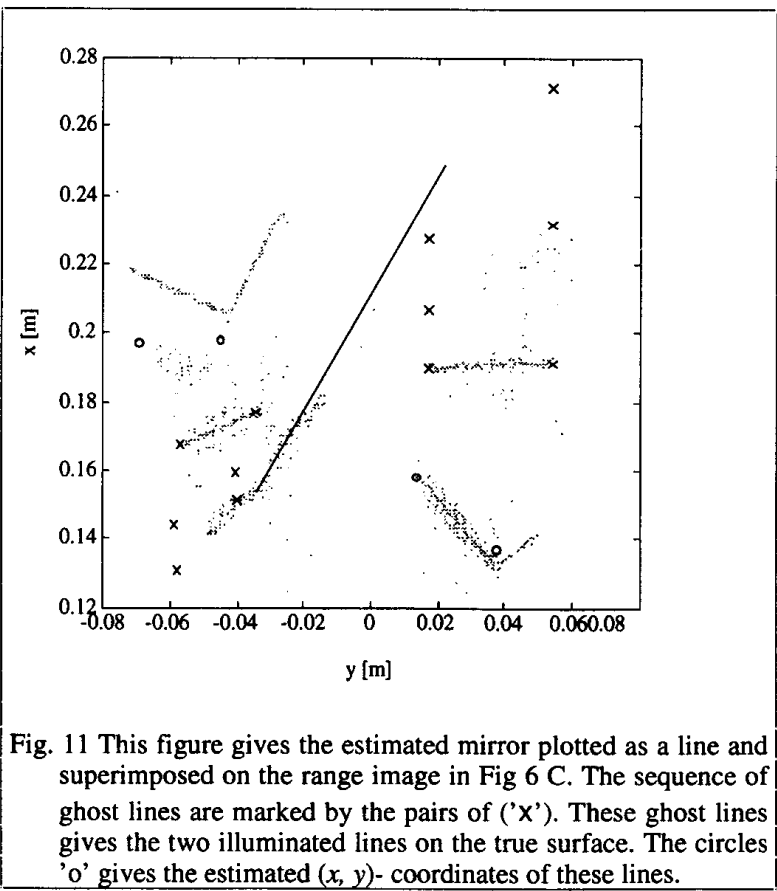

\section{REDUCING THE AMBIGUITIES USING A LIGHT PLANE WITH 19-RAYS}

One inherent weakness with a range camera is the loss of identity along the scan. By using a plane of individual laser rays as shown in Fig 12, the detection and tracking of 'ghosts' is simplified. 
Detecting 'ghosts' is simpler since we can use the additional constraint that an intersection must lie on one of the 19 original rays. It is possible to use epipolar lines [Ho86] to find false points directly in the camera.

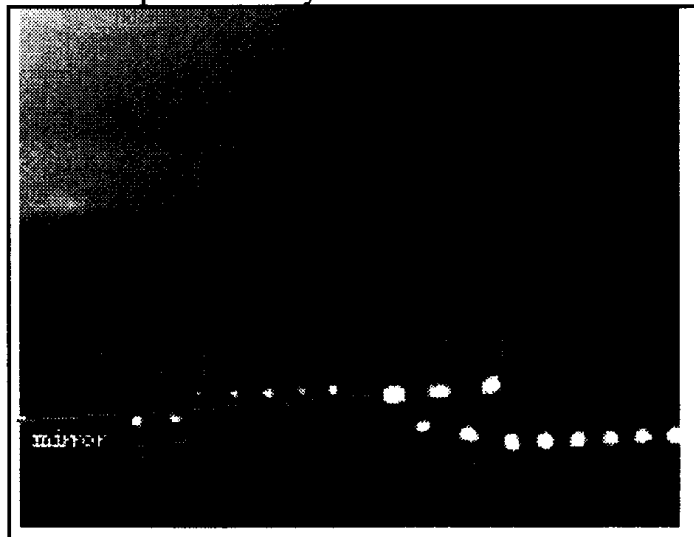

Fig 12 Using a plane of individual laser rays simplifies the detection of 'ghosts' since they usually do not lie on one of the rays. The mirror image 5 and 4 to the left, or the reflected laser 1,2 and 3 to the right obviously do not lie on any of the 19-rays.

Tracking is simplified since each ray is individual not one in a homogenous plane.

However, this method has a larger need of a model of the scene since the position of a ray intersection will generally move when the range camera moves.

To be able to use the geometry in Fig. 10 to find the mirror, a model of the surfaces of the object must be used to compensate for the motion of the rays.

\section{SUMMARY AND FUTURE WORK}

In this paper, experimental examples and analyses were given of several sources of ambiguities in range camera interpretations. Explicit experiments were given for the cases

- Specular scattering (Fig 1, 3, 4, 6, 9 and 12)

- Multiple scatterings (Fig 1, 4, 6, 9 and 11 )

- Internal scattering giving 'glowing parts' (Fig 5)

- Transparent object having specular peaks (the bottle in Fig. 3 )

For scenes with a plane mirror, it was shown that:

- A mirror image for case MOM with light propagation laser $\rightarrow$ Mirror $\rightarrow$ Object $\rightarrow$ Mirror $\rightarrow$ camera can not be distinguished from the basic case $\mathrm{O}$ (laser $\rightarrow$ Object $\rightarrow$ camera) for a sheet-of-light range cam era.

- A reflected laser (case MO) and mirror image (case OM) of a light plane intersection is detectable if the mirror is oriented with a normal vector pointing out of the light plane.

- The position and orientation of the mirror can be estimated if detected 'ghosts' 'span the mirror image' of the light plane.

Current and forthcoming work has been focused on:
- Experiments with other sources of illumination e.g. dots on a line

- Finding a modelling framework for reducing/detecting ambiguities by motion. This includes extension of the analysis above for a scanning laser plane. We also intend to include occlusion effects [MaB93] .

- Analysis of how the shape of the laser plane and extraction method effects range noise is in progress.

- The problem of detecting changes in a scene [KLO93], [MP 71], [BSF 88] is an application as well as feedback laws for gripping [WBN92], [FLÅW93].

\section{APPENDIX}

\section{Reflection of a unit ray}

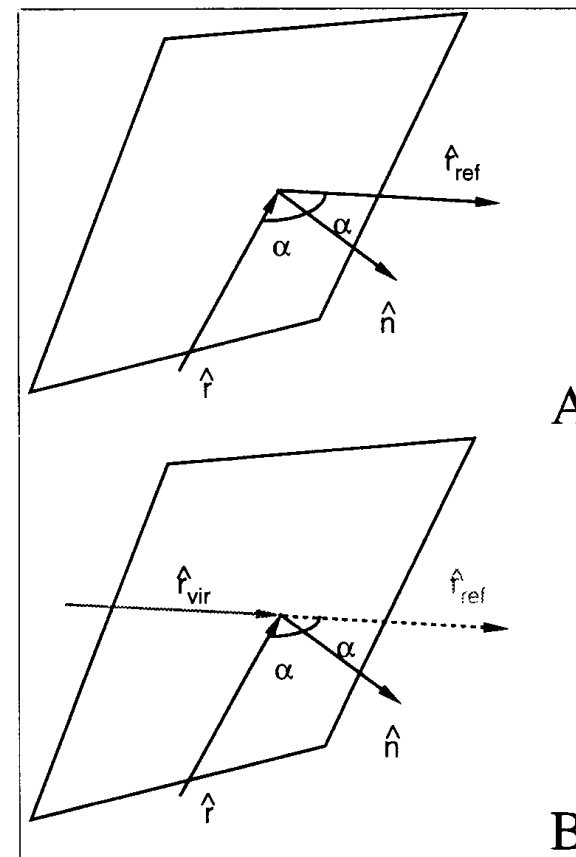

Fig A1: A: Reflection of a unit ray $\hat{r}$ in a mirror with normal $\hat{n}$. B: Virtual image of a unit ray $\hat{r}$ in a mirror with normal $\hat{n}$.

For a perfect reflection Snell's Law must hold:

-a: the vectors $\hat{r}, \hat{n}$ and $\hat{r}_{\text {ref }}$ all belong to the same plane i. e. $\hat{r}_{\text {ref }}=a \hat{r}+b \hat{n}$

-b: the angle between the incoming ray and the normal is equal with the angle between the reflected ray and the normal ( $\alpha$ :s in Fig A1) i. e. $-\hat{r} \bullet \hat{n}=\hat{r}_{\text {ref }} \bullet \hat{n}$

now use $\left(^{*}\right)$ in $(* *)$ :

$-\hat{r} \bullet \hat{n}=(a \hat{r}+b \hat{n}) \bullet \hat{n} \Rightarrow b=-(1+a) \hat{r} \bullet \hat{n}$

but $\hat{r}_{\text {ref }}$ is a unit vector $\Rightarrow \hat{r}_{r e f} \bullet \hat{r}_{\text {ref }}=1 \Rightarrow$ $(a \hat{r}+(-(1+a) \hat{r} \bullet \hat{n}) \hat{n}) \bullet(a \hat{r}+(-(1+a) \hat{r} \bullet \hat{n}) \hat{n})=1 \Leftrightarrow$ 
$a^{2}+(\hat{r} \bullet \hat{n})^{2}\left((1+a)^{2}-2 a(1+a)\right)=1 \Leftrightarrow$

$a^{2}+(\hat{r} \bullet \hat{n})^{2}(1+a)(1-a)=1$

which holds for any $\hat{r} \bullet \hat{n}$ iff $a= \pm 1$

with $a=-1$ as a degenerate case i. e.

$\hat{r}_{r e f}=\hat{r}-((1+1) \hat{r} \bullet \hat{n}) \hat{n}=\hat{r}-2(\hat{r} \bullet \hat{n}) \hat{n}$

Virtual image of a unit ray

As the direction of the virtual image of $\hat{r}$ must be the same as the direction of the reflected ray, it follows directly that

$\hat{r}_{\text {vir }}=\hat{r}-2(\hat{r} \bullet \hat{n}) \hat{n}$

\section{Proof of property 1}

Consider two direction rays $\hat{r}_{1}$ and $\hat{r}_{2}$ in the plane $P$. Their reflected counterparts are:

$\hat{r}_{\text {ref } 1}=\hat{r}_{1}-2\left(\hat{r}_{1} \bullet \hat{n}\right) \hat{n}$ and $\hat{r}_{\text {ref } 2}=\hat{r}_{2}-2\left(\hat{r}_{2} \bullet \hat{n}\right) \hat{n}$

with $A$ as the normal of the mirror.

Now define the normal of the plane $P$ as

$n_{p}=\hat{r}_{1} \times \hat{r}_{2}$ with corresponding normal for $P_{g}$

$n_{P g}=\hat{r}_{r e f 1} \times \hat{r}_{r e f 2}=\left(\hat{r}_{1}-2\left(\hat{r}_{1} \bullet \hat{n}\right) \hat{n}\right) \times\left(\hat{r}_{2}-2\left(\hat{r}_{2} \bullet \hat{n}\right) \hat{n}\right)=$

$\hat{r}_{1} \times \hat{r}_{2}-2\left(\left(\hat{r}_{1} \times \hat{r}_{2}\right) \bullet \hat{n}\right) \hat{n}=n_{P}-2\left(n_{P} \bullet \hat{n}\right) \hat{n}$

For the virtual counterparts to $\hat{t}_{1}$ and $\hat{\imath}_{2}$ we have

$\hat{r}_{\text {vir } 1}=\hat{r}_{1}-2\left(\hat{r}_{1} \bullet \hat{n}\right) \hat{n}$ and $\hat{r}_{\text {vir } 2}=\hat{r}_{2}-2\left(\hat{r}_{2} \bullet \hat{n}\right) \hat{n}$

with normal:

$n_{P v}=\hat{r}_{v i r 1} \times \hat{r}_{\text {vir } 2}=\left(\hat{r}_{1}-2\left(\hat{r}_{1} \bullet \hat{n}\right) \hat{n}\right) \times\left(\hat{r}_{2}-2\left(\hat{r}_{2} \bullet \hat{n}\right) \hat{n}\right)=$

$\hat{r}_{1} \times \hat{r}_{2}-2\left(\left(\hat{r}_{1} \times \hat{r}_{2}\right) \bullet \hat{n}\right) \hat{n}=n_{P}-2\left(n_{P} \bullet \hat{n}\right) \hat{n}=n_{P g}$

Since all planes must intersect with the mirror at the same line, the two planes $P_{g}$ and $P_{v p}$ are identical (except for their extent).

\section{Proof of property 3}

In Fig. A2 we consider the case when the light-plane first hits the a mirror and then a diffuse object. The illuminated line on the object is finally observed by the camera through the mirror. Below we show that the mirror image stays stationary as the robot's motion is confined to the light-plane.

Consider a single ray $\hat{r}_{l} \in P$ reaching the mirror at $\bar{r}_{r 0}$.

The new direction after reflection in the mirror is $\hat{r}_{r}=\hat{r}_{l}-2\left(\hat{r}_{l} \bullet \hat{n}_{M}\right) \hat{n}_{M}$,

where $\hat{n}_{M}$ is the unit normal of the mirror.

This reflected ray illuminates the object at the point

$$
\bar{r}_{p}=\bar{r}_{r 0}+s \hat{r}_{r}=\bar{r}_{r 0}+s\left(\hat{r}_{l}-2\left(\hat{r}_{l} \bullet \hat{n}_{M}\right) \hat{n}_{M}\right)
$$

where $s$ is the distance along the ray $\hat{r}_{r}$ from $\bar{r}_{r 0}$ to $\bar{r}_{p}$.

This illuminated point has the virtual image

$\bar{r}_{v}=\bar{r}_{r 0}+s \hat{r}_{r}-2\left|\left(\bar{r}_{r 0}-\left(\bar{r}_{r 0}+s \hat{r}_{r}\right)\right) \cdot \hat{n}_{M}\right| \hat{n}_{M}$

when seen in the mirror.

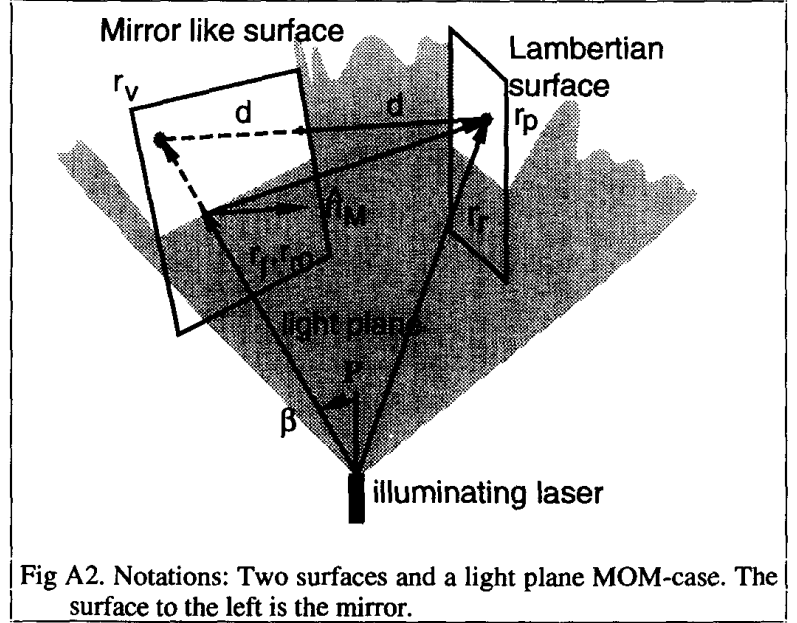

Substitute $\hat{r}_{r}$ and use $\hat{r}_{l} \bullet \hat{n}_{M}<0$ for a visible surface with $\hat{n}_{M}$ pointing out of the surface

$$
\begin{aligned}
& \bar{r}_{v}=\bar{r}_{r 0}+s \hat{r}_{l}-2 s\left(\hat{r}_{l} \bullet \hat{n}_{M}\right) \hat{n}_{M} \\
& -2\left|-s\left(\hat{r}_{l}-2\left(\hat{r}_{l} \bullet \hat{n}_{M}\right) \hat{n}_{M}\right) \bullet \hat{n}_{M}\right| \hat{n}_{M}= \\
& \quad=\bar{r}_{r 0}+s \hat{r}_{l}-2 s\left(\hat{r}_{l} \bullet \hat{n}_{M}\right) \hat{n}_{M}+2 s\left(\hat{r}_{l} \bullet \hat{n}_{M}\right)
\end{aligned}
$$

thus

$\bar{r}_{v}=\bar{r}_{r 0}+s \hat{r}_{l}$

hence the virtual image lies is in the same direction as the original laser ray and the plane spanned by the virtual rays is the same as the original plane $P$.

Since the virtual point lies on an original ray it will behave just as a real point at the same position. The same applies to any MOM-intersection, for example the rear corner of the box in Fig 6.

\section{Reflection in a polished cylinder}

For a mirror like cylinder the reflected surfaces will no longer be planar. In this section expressions for the reflected surface $C_{g}$ corresponding to $P_{g}$ for the planar mirror are presented. Let the reflecting cylinder of radius $R$ be defined by the direction of its symmetry-axis $\hat{n}_{c}$ and one arbitrary point $\bar{r}_{c}$ on the centre axis.

The intersection between $P$ and the cylinder will be an ellipse. The surface normal of the cylinder at an illuminated point $\bar{r}_{P c}$ is

$$
\hat{n}=\frac{\left(\left(\bar{r}_{p_{c}}-\bar{r}_{c}\right) \bullet \hat{x}_{c}\right) \hat{x}_{c}+\left(\left(\bar{r}_{p_{c}}-\bar{r}_{c}\right) \bullet \hat{y}_{c}\right) \hat{y}_{c}}{\left|\left(\left(\bar{r}_{p_{c}}-\bar{r}_{c}\right) \bullet \hat{x}_{c}\right) \hat{x}_{c}+\left(\left(\bar{r}_{P c}-\bar{r}_{c}\right) \bullet \hat{y}_{c}\right) \hat{y}_{c}\right|} .
$$

With $\hat{x}_{c}, \hat{y}_{c}$ as any orthogonal vector pair spanning the plane with normal $\hat{n}_{c}$.

Using this surface normal and all illuminated points $\tilde{r}_{l c}$ we can compute the surface of the reflected laser-plane as the surface starting at $\bar{r}_{l c}$ and extending in direction $C_{g}$. 

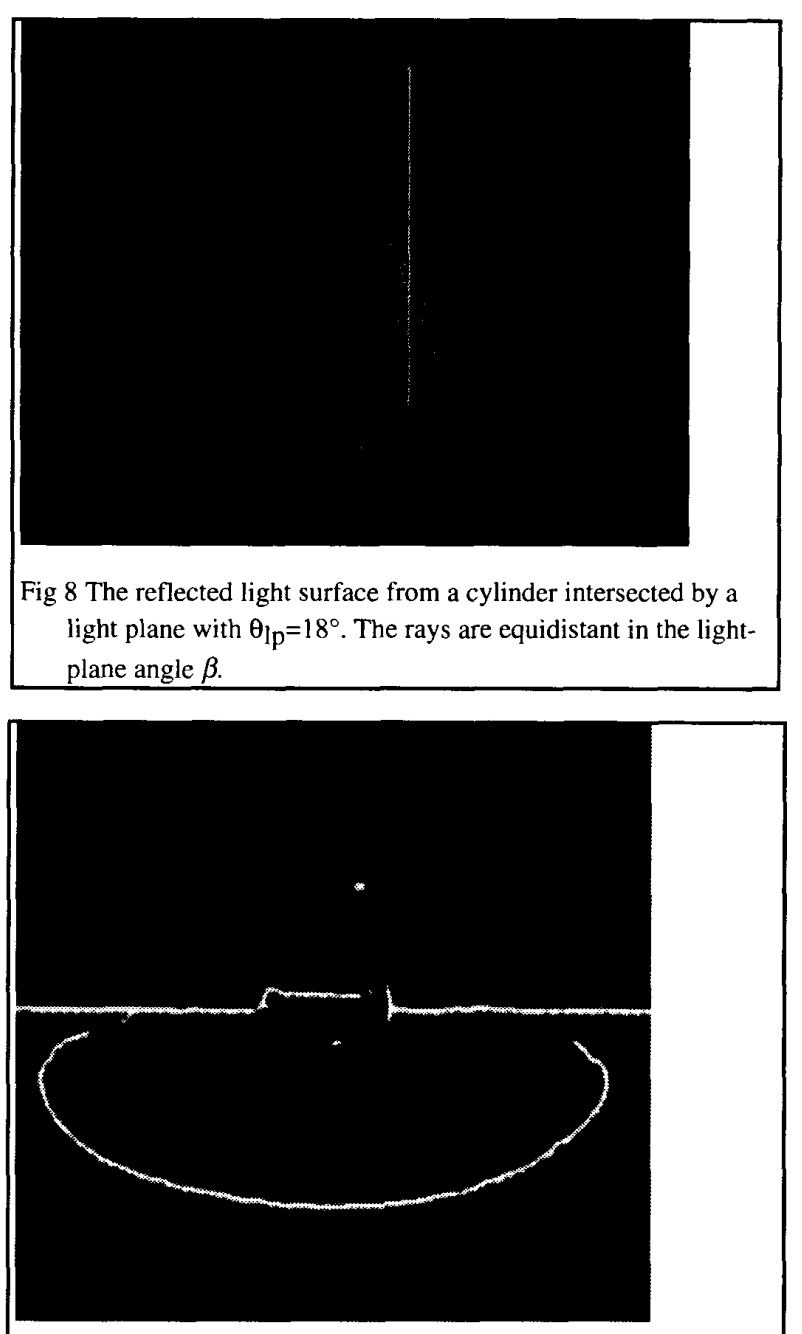

Fig 9 A polished steel cylinder illuminated by a light-plane with incidence angle 14 degrees. Notice that the reflected rays reaching the floor has larger intensity than direct illumination on the cylinder. An exception is the specular blob. The line on the cylinder is the mirror image of the curve on the floor! (the image has been enhanced to survive in printing).

$C_{g}=\left\{\hat{r}_{s}\right\}$, where $\hat{r}_{s}=\hat{r}_{p}-2\left(\hat{r}_{p} \bullet \hat{n}\right) \hat{n}$,

using $\hat{r}_{p}=\left[\cos (\beta) \cos \left(\theta_{l p}\right), \sin (\beta),-\cos (\beta) \sin \left(\theta_{l p}\right)\right]$ as the direction of the ray in $P$ that hits the cylinder at $\bar{r}_{p_{\mathrm{c}}}$.

Note in Fig 8 that this surface no longer is a plane. From these rays it is then possible to continue with their intersections with other surfaces in a way similar to subsection V-A. As an example the surface in Fig 8 is calculated for an inter section with a floor-plane. Compare this with the ring of light on the floor in Fig 9.
With a scanning motion upwards of the light-plane, the curved arc on the floor will move out from the cylinder. This implies a surface which would have occluded the prior laser scan line. If not transparent objects are allowed such motion is inconsistent (for a range camera based on assumption of light only from plane $P$ ).

\section{ACKNOWLEDGMENT}

The authors thanks P. Kirkegaard, B. Nilsson and the reviewers for their valuable comments.

\section{REFERENCES}

[Bla85] Blake, A Specular stereo Proc. IJCAI Conf. Los Angeles CA, USA, 1985

[ZGB89] Zisserman, A., Giblin, P., Blake, A. The information available to a moving observer from specularities, Image and Vision Computing Vol. 7 No. 1. pp 38-42, 1989

[MP 71] Mehra R. K. Peschon J. An Innovations Approach to Fault Detection and Diagnosis in Dynamic Systems, Automatica Vol. 7 pp 637-640,1971

[BSF 88] Bar-Shalom Y. Fortmann T. E. Tracking and Data Association, Academic Press Vol. 179, 1988

[NIK91] Nayar S.K., Ikeuchi K., Kanade T., Surface Reflection: Physical and Geometrical Perspectives, IEEE Transactions on Pattern Analysis and Machine Intelligence (1991), vol. 13, No. 7

[KLO93] Ljunggren-Klöör P. Lundquist P. Ohlsson P. Nygårds J. and Wernersson $\AA$., Change Detection in Natural Scenes using Laser Range Measurements from a Mobile Robot, IAV Southampton April 1993 pp 71-76

[WBN92] Wernersson A., Boberg B., Nilsson B., Nygårds J. and Rydberg T., On gripping using range cameras: sensor noise, task tolerances and feedback, Proc. Robotics and Automation 1992, pp. 1654-1660.

[Ho86] Horn B.K.P, Robot Vision. MIT Press (1986),

[FLÅW93] Forsberg J, Larsson U, Åhman P and Wernersson $\AA$ (1993) The Hough Transform inside the Feedback Loop of a Mobile Robot, Proc. Robotics and Automation 1993, Vol 1 pp. $791-798$.

[NW 93] Nygårds J. and Wernersson A. Gripping Using a Range Camera: Reducing Ambiguities for Specular and Transparent Objects Robotics workshop in Linköping 1993 [AcH88] Acharya P K and Henderson T C Parameter Estimation and Error Analysis of Range Data, Proc. Robotics and Automation 1988

[MaB93] Maver J. and Bajcsy R. Occlusions as a Guide for Planning the Next View, IEEE Trans. Pattern Analysis and Machine Intelligence, May 1993

[DaX91] Danielsson P.-E. Xu S. Depth From Ego-Motion and Optic Flow, 6th Int. Conf. on Image Analysis and Processing, Como, Italy, September, 1991.

[Wo194] Wolff, L. B., Relative brightness of specular and diffuse reflection, Optical Engineering Vol. 33 No. 1 pp. 285-293, January 1994. 